\title{
COMPREHENSIVE ASSESSMENT OF ANTIOXIDANT AND CHELATING CAPACITY OF SOME BIOGENIC AMINES AND RELATED DRUGS
}

\author{
ALEXANDRINA GUIDEA ${ }^{\mathrm{a}}$, AUGUSTIN C. MOTु \\ COSTEL SÂRBU ${ }^{*}$
}

\begin{abstract}
The radical scavenging, reducing power and metal-chelating capacity of some biogenic amines and related sympathomimetic drugs were clarified using various in vitro antioxidant assays as DPPH (1, 1-diphenyl-2picryl-hydrazyl), ABTS (2,2'-azino-bis (3-ethylbenzthiazoline-6-sulfonic acid), SORS (superoxide anion $\left(\mathrm{O}_{2}{ }^{-}\right)$radical-scavenging), nitric oxide (NO) radical scavenging, FRAP (ferric reducing antioxidant power), CUPRAC (cupric reducing antioxidant capacity) and CHROMAC (chromium reducing antioxidant capacity), including also FIC (ferrous ion-chelating capacity). The highest radical scavenging values in the case of DPPH test, for example, were obtained for methyldopa $(93.14 \%)$, isoprenaline $(92.92 \%)$ adrenalone $(90.76 \%)$ and dopamine $(90.51 \%)$. The highest reduction power, according to FRAP test, presented the same compounds: adrenalone $(96.02 \%)$ methyldopa $(95.97 \%)$, dopamine (94.67\%) and isoprenaline (93.72\%), except metaraminol (5.79\%). Concerning the chelating capacity, adrenalone $(66.35 \%)$, metaraminol (55.31\%), metaproterenol $(49.58 \%)$ and terbutaline $(45.64 \%)$ showed the higher chelating capacity. The lowest value, in this case, was obtained for methyldopa $(0.28 \%)$. According to the results obtained in the present study, the investigated drugs showed an effective in vitro antioxidant and radical scavenging ability and metal-chelating capacity. In addition, applying hierarchical cluster analysis (HCA), principal component analysis (PCA) and the sum of ranking differences (SRD) similarities and differences of investigated compounds and considered assays were clearly proved.
\end{abstract}

Keywords: Antioxidant and chelating capacity, biogenic amines, sympathomimetic drugs, chemometrics

a Faculty of Chemistry and Chemical Engineering, Department of Chemistry, Babeș-Bolyai University, 400028, Cluj-Napoca, Romania

*Corresponding author: csarbu@chem.ubbcluj.ro; Tel: (+40-264-59383) 


\section{INTRODUCTION}

An antioxidant is a chemical that prevents the oxidation of other chemicals. According to Halliwell and Gutteridge [1, 2] an antioxidant is „any substance that delays, prevent or removes oxidative damage to a target molecule". They protect the key cell components by neutralizing the damaging effects of free radicals, which are natural byproducts of cell metabolism. The free radicals attack the nearest stable molecules, stealing their electrons. When the attacked molecule loses its electrons, it becomes a free radical itself, beginning a chain reaction, finally resulting in the deterioration of a living cell. To protect the cells from the damage caused by oxidants, the organisms have evolved several antioxidant defense mechanisms for the rapid and efficient removal of reactive oxygen species (ROS) or reactive nitrogen species (RNS) from the intracellular environment $[3,4]$. In normal circumstances, there is a balance between antioxidants and oxidants. When the equilibrium between oxidants and antioxidants defense systems is imbalanced in favor of the oxidants, the condition is known as oxidative stress. Oxidative stress results in the damage of biopolymers including nucleic acids, proteins, polyunsaturated fatty acids and carbohydrates leading to a variety of human diseases like Alzheimer's disease, Parkinson's disease, atherosclerosis, cancer, arthritis, immunological incompetence and neurodegenerative disorders [5].

Biogenic Amines (BAs) are known to occur in all living organisms (microorganisms, plants, and animals). BAs are the basic nitrogenous compounds with aliphatic, (putrescine, cadaverine, spermine, spermidine), aromatic (tyramine, phenylethylamine), or heterocyclic (histamine, tryptamine) structure. The biogenic amines derived from amino acid tyrosine are called catecholamines because they contain a catechol or 3,4-dihydroxylphenyl group. In the human body, the most abundant catecholamines are epinephrine (adrenaline), norepinephrine (noradrenaline) and dopamine. Catecholamines are hormones released by the sympathetic nervous and adrenal medulla in response to a range of stresses to regulate the host physiological functions in living systems [6-10]. As it is also demonstrated, catecholamines are able to act as antioxidants or prooxidants depending on various factors such as concentration, $\mathrm{pH}$, the test system used for investigation (in vitro and in vivo) or possible interaction with other antioxidants, different substrates or with transition metal ions [11-13]. The high antioxidant potential of catecholamine drugs is attributed to the fact that the semiquinone radical derived from $\mathrm{H}$-atom donation of catechol can be stabilized by an intramolecular hydrogen bond and the electron-donating properties of the ortho-OH [11-15].

Since ROS and RNS are implicated in the pathogenesis of many chronic diseases, as has been shortly mentioned above, finding natural and synthetic antioxidants to combat ROS and RNS has attracted much attention [16-19]. 
Among the therapeutics with promising antioxidant activity, adrenergic drugs (agonists and antagonists - blockers) are of a large interest because they are considered the future medication for new improvements in different therapies $[17,20]$. The beneficial effects shown by some adrenergic compounds in neuroprotection and in experimental models of Parkinson's disease, for example, has already been associated with their antioxidant properties and the current studies are oriented on the potential future use of these drugs in the treatment of various diseases as osteoporosis, cancer and malaria. Considering all these aspects, a better knowledge of the antioxidant profile of redox-active drugs will involve different investigations for increasing bioavailability, safety, and efficiency of future improved therapeutics.

In vitro antioxidant activity assays show extreme diversity concerning the distinct oxidation step, the measurement of the outcome and the mode of expressing the antioxidant activity results [21]. In the above considerations, the radical scavenging ability and reducing power capacity of some biogenic amines and related sympathomimetic drugs were investigated using various in vitro antioxidant assays including DPPH (1, 1-diphenyl-2-picryl-hydrazyl), ABTS (2,2'-azino-bis (3-ethylbenzthiazoline-6-sulfonic acid), SORS (superoxide anion $\left(\mathrm{O}_{2}^{-}\right)$radical-scavenging assay), nitric oxide (NO) radical scavenging assay, FRAP (ferric reducing antioxidant power), CUPRAC (cupric reducing antioxidant capacity) and CHROMAC (chromium reducing antioxidant capacity), including also FIC (ferrous ion-chelating capacity) [22-25]. The results obtained allow a relevant comparison of the investigated compounds concerning their radical scavenging, reducing power and ion-chelating capacity and an objective comparison of the antioxidant assays applying various chemometric methods.

\section{RESULTS AND DISCUSSION}

According to previous study [24], some important compounds (as biogenic amines and their related drugs) involved in usual metabolic processes proved to have effective in vitro radical scavenging activity based on DPPH assay. For a comprehensive evaluation of their radical activity profiles, the usage of several methods with different mechanisms, kinetics and oxidant species combined with chemometric methods was proposed and evaluated.

The results (mean \pm standard deviation) obtained in this study for the investigated compounds presented in Table 1 are shown in Table 2 and Figure $1 \mathrm{a}-\mathrm{b}$ and Figure 2.

It can be easily observed from the box and whiskers plot that when a robust confidence interval is considered (Figure 1a) the outliers and extremes 
values are not highlighted, and the distributions appear more or less asymmetric. When the classical confidence interval for mean is considered $(p=0.95)$ some outliers and few extremes values are highlighted (Figure 1b).

Table 1. Name, chemical structure and pharmacological activity of investigated compounds

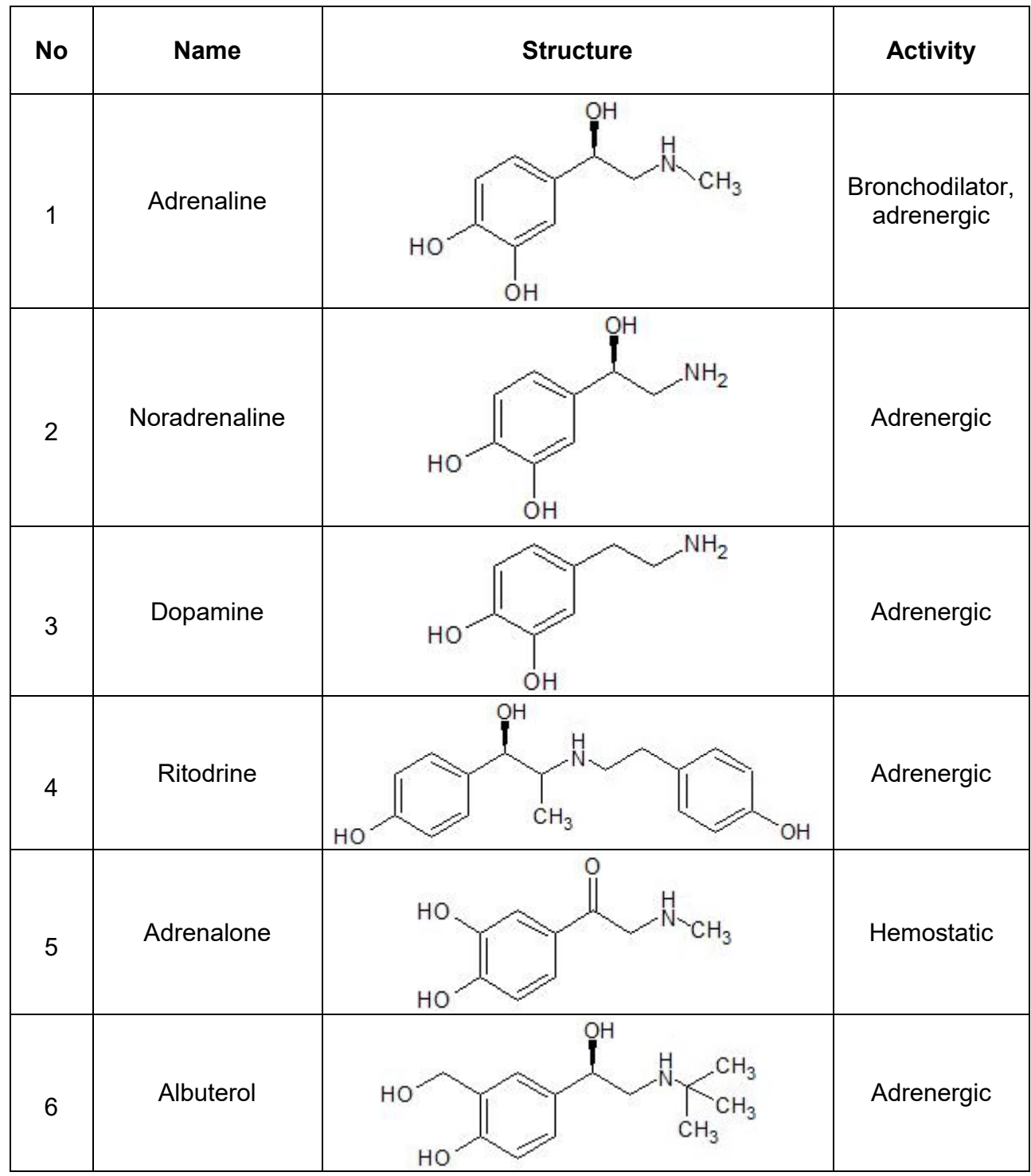


COMPREHENSIVE ASSESSMENT OF ANTIOXIDANT AND CHELATING CAPACITY OF SOME BIOGENIC AMINES AND RELATED DRUGS

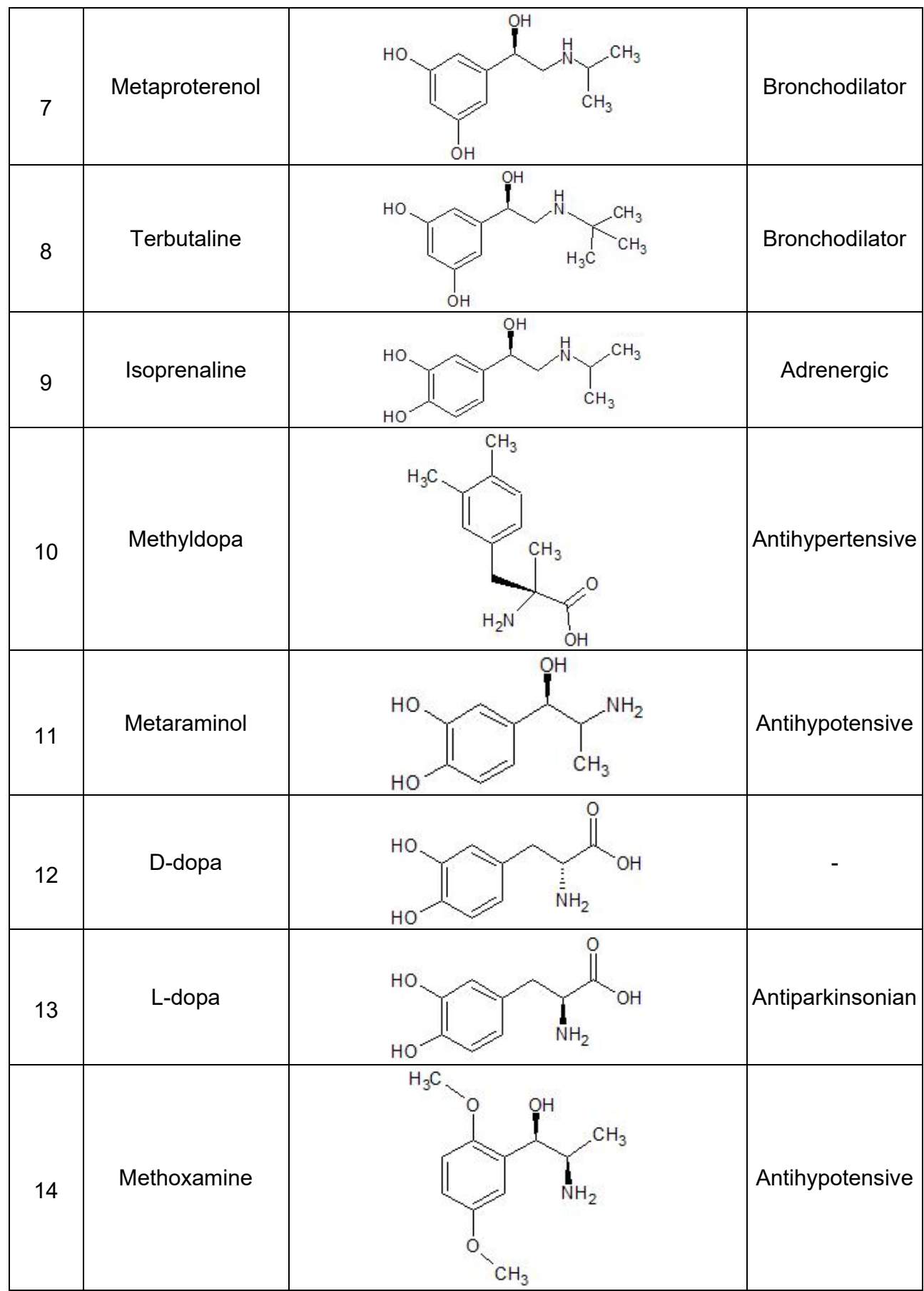




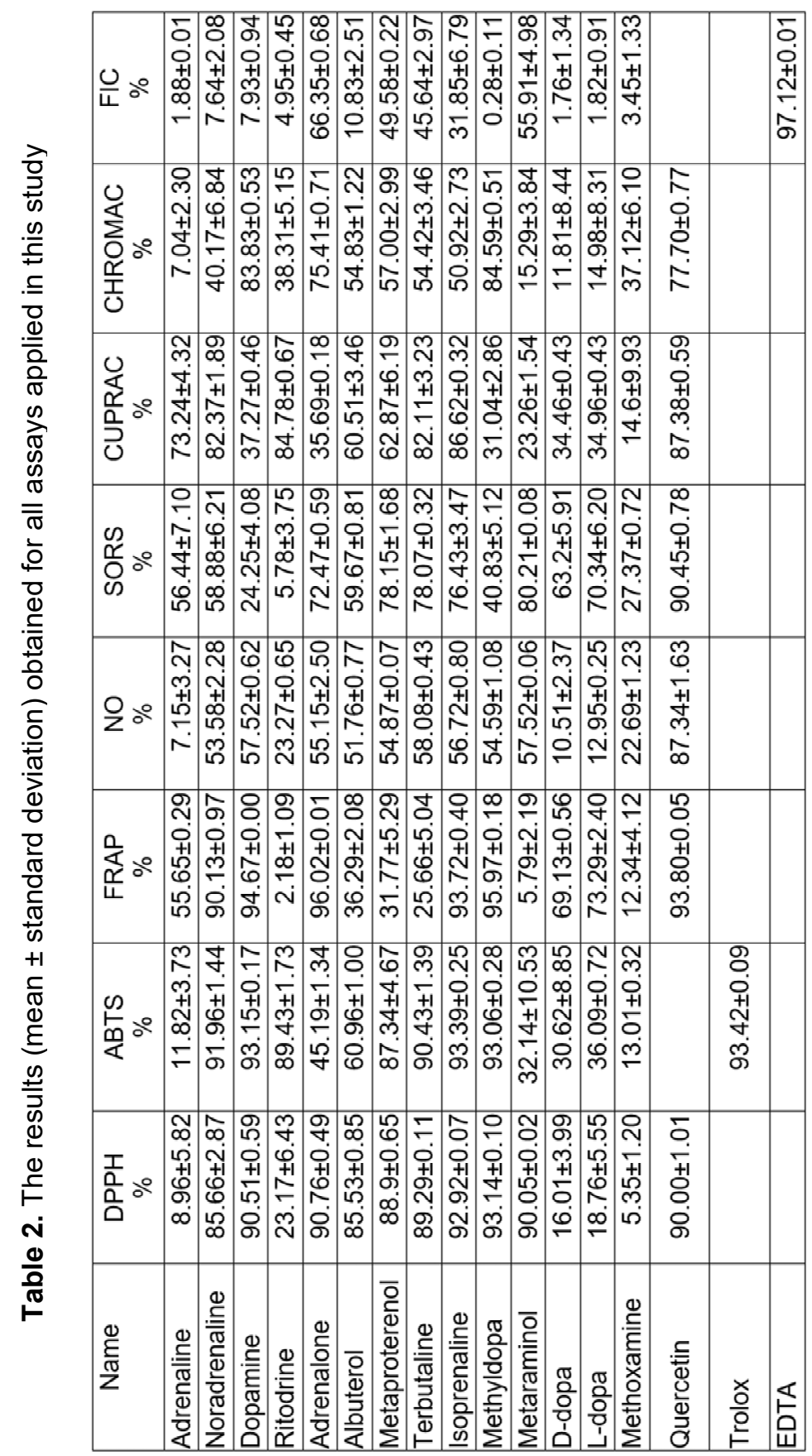


OF SOME BIOGENIC AMINES AND RELATED DRUGS

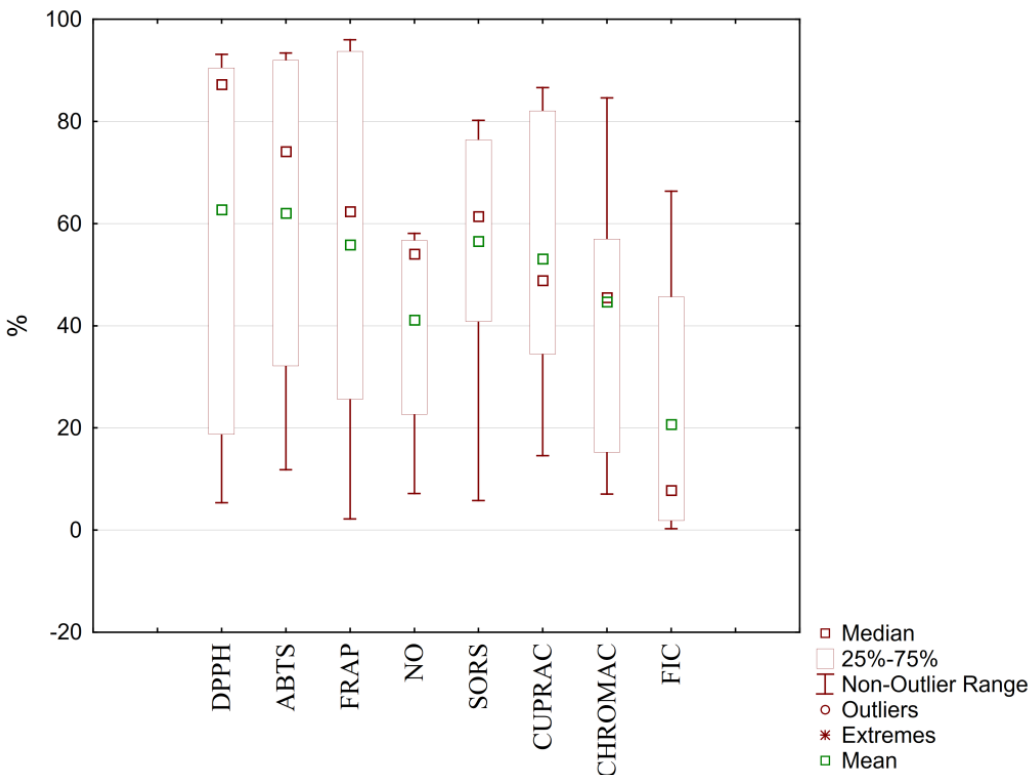

(a)

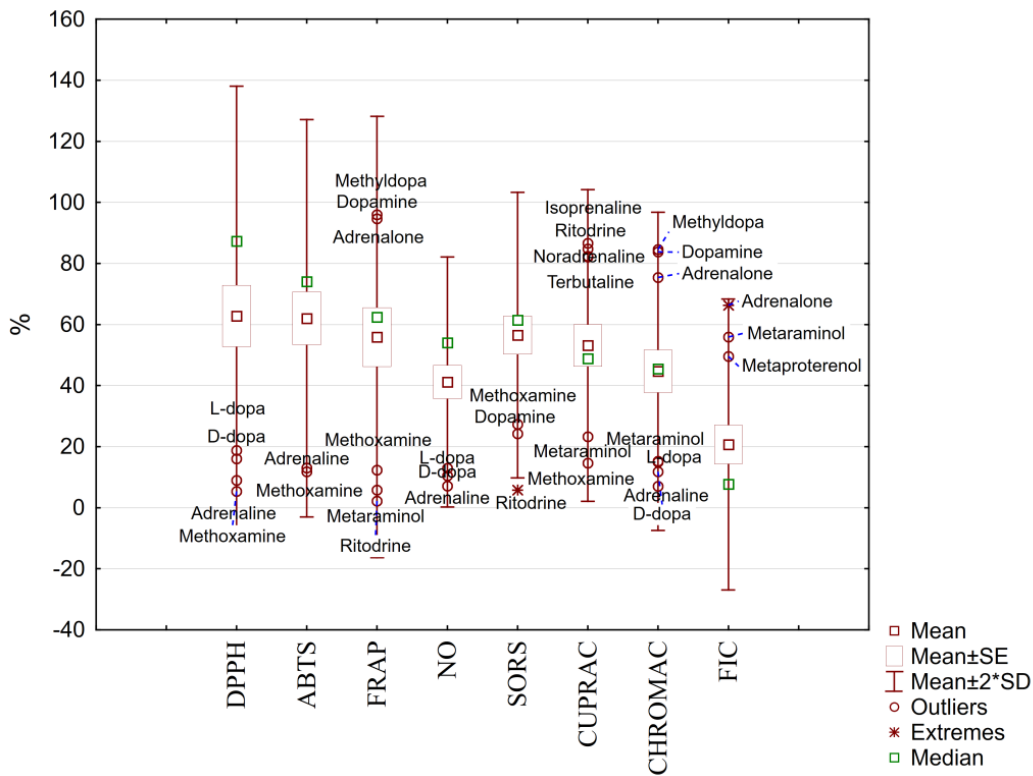

(b)

Figure 1. Box and whiskers plot: robust confidence interval (a), classical confidence interval for mean (b) 
By a carefully examination of the heat map (based on the intensity of colors) in Figure 2 it is interesting to remark that majority of adrenergic drugs have a highest antioxidant activity, except for Methoxamine, D-dopa and Ldopa. In the case of catecholamines, the obtained results for noradrenaline are much more similar to dopamine and adrenergic drugs, but they are quite different from adrenaline.

Radical scavenging ability of biogenic amines and their related drugs was estimated using four methods that implies two synthetic radicals (DPPH and $A B T S$ ) and two free radicals that play as a stress signalling molecules under certain physiological and pathological conditions $\left(\mathrm{O}_{2}{ }^{-}\right.$and $\left.\mathrm{NO}\right)$. Based on obtained results, the samples were grouped into three well defined classes: the group with the highest values for the majority of assays - metaproterenol, terbutaline, isoprenaline and noradrenaline; the group with at least three very high values - adrenalone, albuterol, metaraminol, dopamine, L-dopa, D-dopa and methyldopa and one group with the smallest values - adrenaline, ritodrine and methoxamine. By a comparative evaluation, the catecholamine related drugs (metaproterenol, terbutaline, isoprenaline) are more active than quercetin, in a good concordance with the results reported in the previous study [24] using only the DPPH method.

Considering now the results obtained by reducing antioxidant power assays also we can find some groups with very close values: noradrenaline and isoprenaline; dopamine, adrenalone, methyldopa; albuterol, metaproterenol, terbutaline; L-dopa and D-dopa; metaraminol, methoxamine with the smallest values for all these assays. Adrenaline and ritodrine appear again quite different. The highest reduction power, according to FRAP test, presented the same compounds as above: adrenalone (96.02\%) methyldopa (95.97\%), dopamine $(94.67 \%)$ and isoprenaline (93.72\%), except metaraminol (5.79\%).

Metal chelating capacity of the studied compounds shows a low $\mathrm{Fe}^{2+}$ -chelating ability, except for adrenalone (66.35\%), metaraminol (55.31\%), metaproterenol $(49.58 \%)$ and terbutaline $(45.64 \%)$ which showed the higher chelating capacity. The lowest value, in this case, was obtained for methyldopa $(0.28 \%)$.

These results are very well supported by the graphical representation heat map (Figure 2) in combination with the two dendrograms (obtained applying the HCA method, using the complete method as linkage procedure and Euclidean distance as a measure of similarity in both cases), where the similarity and differences amongst the investigated compounds and the assays can be observed simultaneously. 

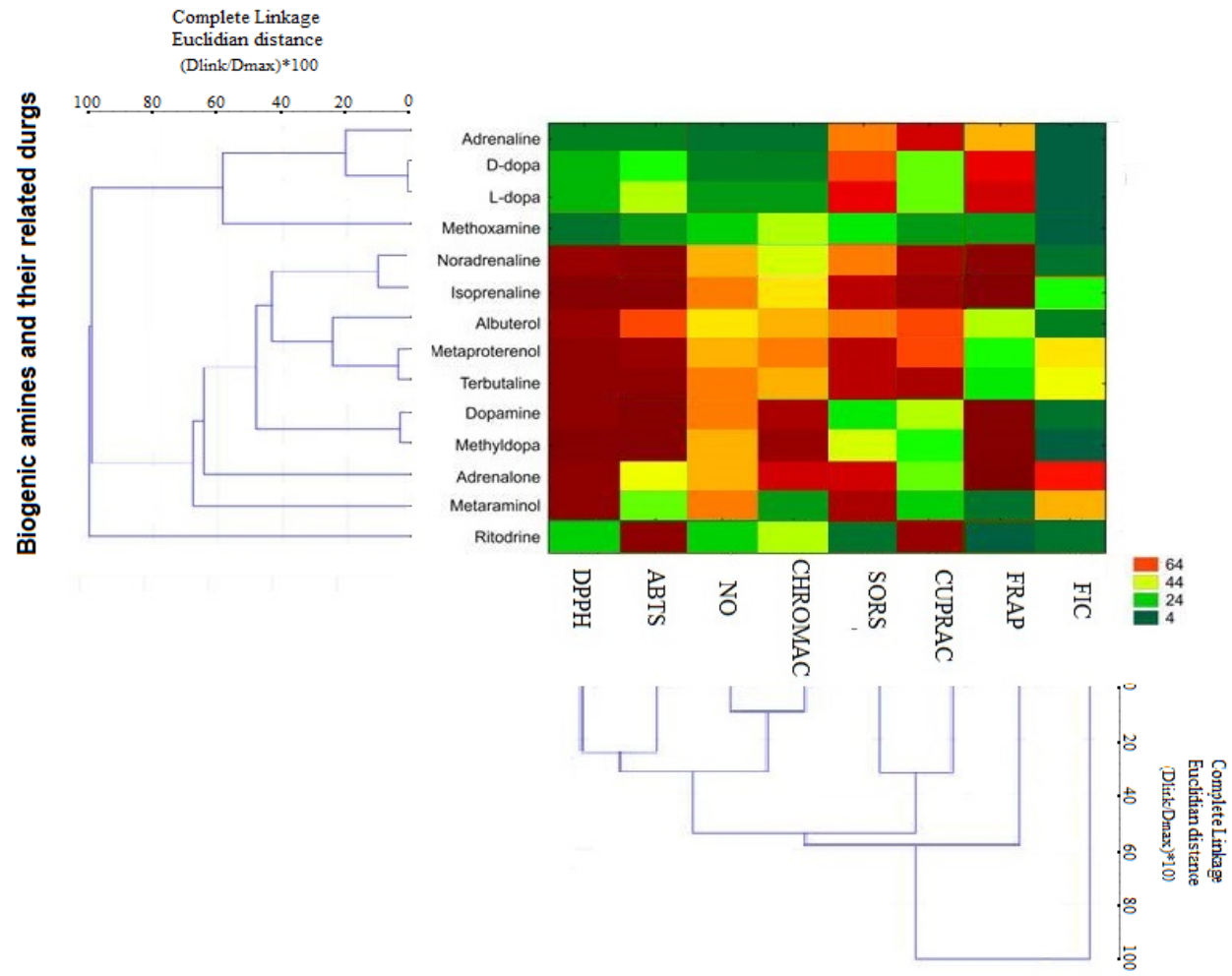

Antioxidant activity assays

Figure 2. The heat map corresponding to all investigated compounds and all assays coupled with hierarchical cluster analysis

The results obtained from principal component analysis (PCA) using the raw data matrix (14 samples and 8 assays) indicate a significant reduction in the number of variables, in good agreement with the correlations shown in Table 3. The first three components explain $84.33 \%$ of the total variation, the first two components explaining $68.18 \%$ from total variance, and the first one only $48.80 \%$. However, the $2 \mathrm{D}$ representation of the scores corresponding to the first two components (2D) or the first three components (3D) indicates a satisfactory separation of compounds according to their similarity (Figure 3a).

Also, the variables (assays) grouping in the space described by the loadings corresponding to the first three principal components confirms once more the similarity and differences established by correlation matrix and hierarchical cluster analysis (Figure $3 b$ ). 
Table 3. Correlation matrix obtained for all assays and compounds

\begin{tabular}{|l|r|r|r|r|r|r|r|r|}
\hline \multirow{2}{*}{ Variable } & \multicolumn{7}{|c|}{ Marked correlations are significant at $p<.05000$} \\
\cline { 2 - 9 } & DPPH & ABTS & FRAP & NO & SORS & CUPRAC & CHROMAC & FIC \\
\hline DPPH & $\mathbf{1 . 0 0 0}$ & $\mathbf{0 . 6 6 4}$ & 0.302 & $\mathbf{0 . 9 7 5}$ & 0.380 & 0.160 & $\mathbf{0 . 6 9 1}$ & $\mathbf{0 . 5 8 6}$ \\
\hline ABTS & & $\mathbf{1 . 0 0 0}$ & 0.251 & $\mathbf{0 . 6 5 7}$ & -0.115 & $\mathbf{0 . 5 3 7}$ & $\mathbf{0 . 6 6 6}$ & 0.099 \\
\hline FRAP & & & $\mathbf{1 . 0 0 0}$ & 0.183 & 0.136 & -0.023 & 0.396 & -0.135 \\
\hline NO & & & & $\mathbf{1 . 0 0 0}$ & 0.283 & 0.124 & $\mathbf{0 . 7 1 9}$ & $\mathbf{0 . 6 0 1}$ \\
\hline SORS & & & & & $\mathbf{1 . 0 0 0}$ & 0.078 & -0.197 & $\mathbf{0 . 6 2 6}$ \\
\hline CUPRAC & & & & & & $\mathbf{1 . 0 0 0}$ & -0.014 & 0.027 \\
\hline CHROMAC & & & & & & & $\mathbf{1 . 0 0 0}$ & 0.224 \\
\hline FIC & & & & & & & & $\mathbf{1 . 0 0 0}$ \\
\hline
\end{tabular}

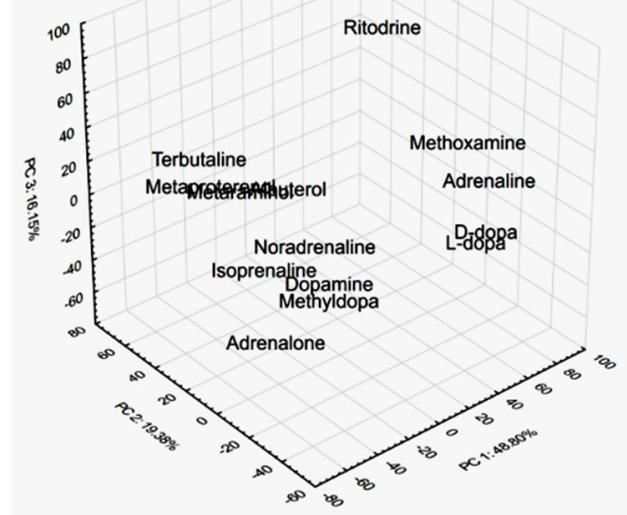

(a)

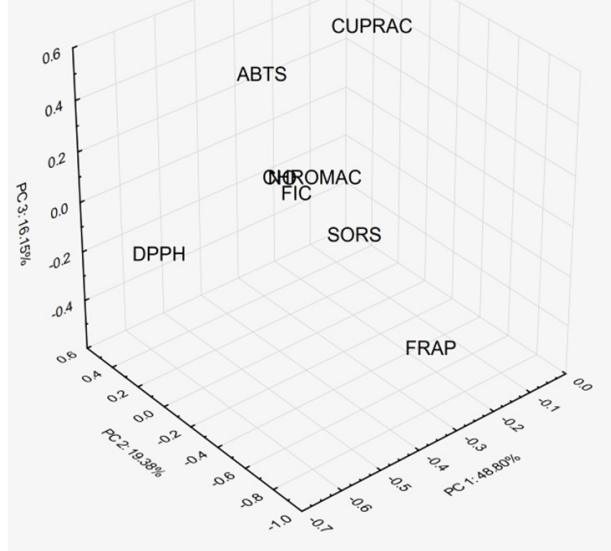

(b)

Figure 3. 3-D score scatterplot of investigated compounds (a) and 3-D loading scatterplot corresponding to all assays used in this study (b)

According to SRD, the best method to express antioxidant activity of investigated biogenic amines and their related drugs is DPPH because it is the method closest to the "average antioxidant and chelating capacity" (the methods appearing on the left side of the Gaussian curve are most similar to the "average antioxidant activity"; thus, the performance of the presented methods should be representative of the final antioxidant behaviour of 
investigated compounds ranks), while the FRAP method is the most different. The results are well illustrated in Figure 4 . We have also to remark the high similarity of the assays clustering with the dendrogram obtained applying HCA.

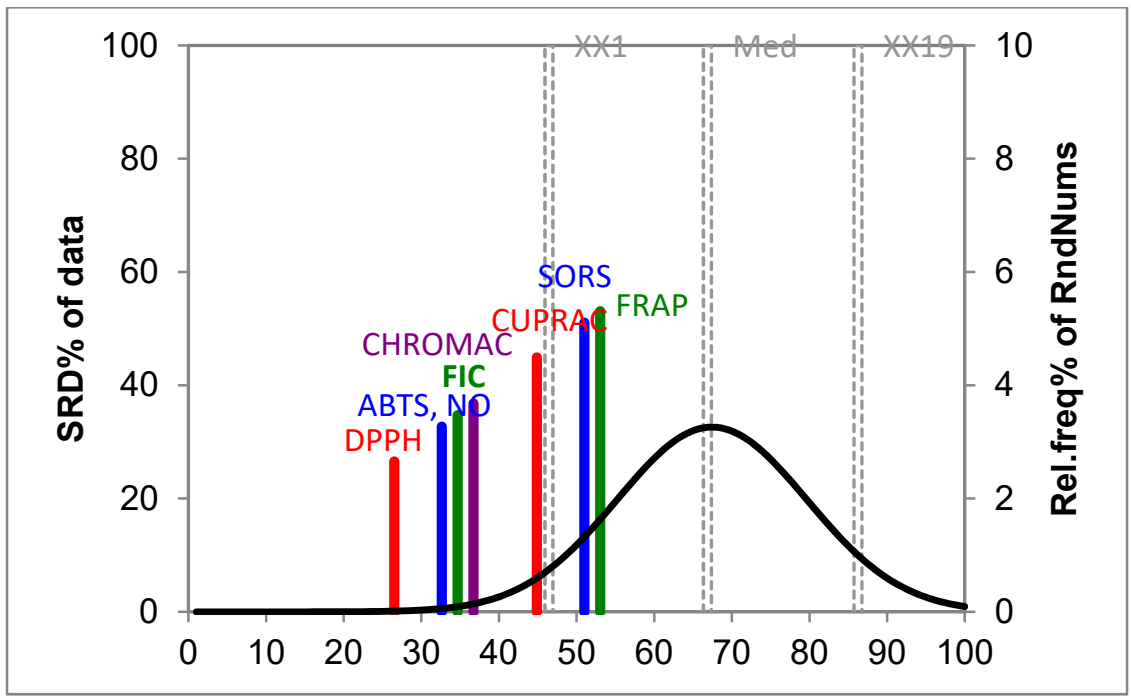

Figure 4. Evaluation of the eight assays using the sum of ranking differences. Average was used as a golden standard. Scaled SRD values are plotted on the $x$-axis and left $y$-axis, the right $y$-axis shows the relative frequencies (black curve).

Parameters of the Gaussian fit: $m=67 \mathrm{~s}=10.3$ Probability levels $5 \%(X X 1)$, Median (Med), and 95\% (XX19) are also given.

\section{CONCLUSIONS}

In this study, applying several in vitro methods including mostly radical scavenging (DPPH, ABTS, SORS, NO) and reducing power (FRAP, CUPRAC, CHROMAC) assays, the high radical scavenging and reducing power capacity of some biogenic amines and related drugs were clearly demonstrated. In addition, the majority of the investigated drugs had shown a moderate ferrous chelating capacity. Moreover, applying hierarchical cluster analysis (HCA), principal component analysis (PCA) and the sum of ranking differences (SRD), similarities and differences of investigated compounds and considered assays were clearly proved. 


\section{EXPERIMENTAL SECTION}

\section{Chemicals and samples preparation}

The biogenic amines investigated in this study include adrenaline, noradrenaline, dopamine, and related drugs: methyldopa, L-dopa, D-dopa, metaraminol, ritodrine, adrenalone, albuterol, metaproterenol, terbutaline, isoprenaline, and methoxamine of analytical grade obtained from Merck and Sigma (Sigma-Aldrich $\mathrm{GmbH}$, Sternheim, Germany). All other reagents were either of analytical grade or of the highest quality available.

The stock solutions of all compounds, including positive controls (quercetin and trolox) were prepared in absolute ethanol (96\%) at concentration $1 \mathrm{mM}$. EDTA solution was prepared in water at concentration $1 \mathrm{mM}$. For those related drugs that were poorly soluble in ethanol (adrenaline, D-dopa), stoichiometric hydrochloric acid was added. The final concentration of investigated compounds ( $300 \mu \mathrm{L})$ was: $1.67 \mu \mathrm{M}$ for DPPH, ABTS, SORS, FRAP, CUPRAC, FIC assays; $20 \mu \mathrm{M}$ for NO assay and $16.67 \mu \mathrm{M}$ for CHROMAC assay. The same concentrations are for reference antioxidant solutions.

\section{Radical scavenging assays}

The DPPH test was carried out according to the method described by Blois [26] with slight modifications. Briefly, $295 \mu \mathrm{L}$ of $0.15 \mathrm{mM} \mathrm{DPPH}^{*}$ prepared in absolute ethanol was added to $5 \mu \mathrm{L}$ of samples in 96 well cuvettes. The mixture was shaken and incubated for $30 \mathrm{~min}$, in dark, at room temperature. Then, the absorbance was recorded at $517 \mathrm{~nm}$, using a microplate reader (Tecan Spark ${ }^{\mathrm{TM}}$ 10M, Männedorf, Switzerland). All determinations were performed in duplicate. Ethanol and quercetin were used as a negative and positive control, respectively.

The ABTS cation radical scavenging capacity was determined according to the method developed by $\mathrm{Re}$ et al. [27]. The $\mathrm{ABTS}^{*}$ cation radical was generated by adding ABTS stock solution $(7 \mathrm{mM})$ into potassium persulfate solution $(2,45 \mathrm{mM})$, and the mixture was incubated in dark for $16 \mathrm{~h}$ at room temperature. The generated $\mathrm{ABTS}^{*+}$ cation radical was diluted with phosphate buffer ( $10 \mathrm{mM}, \mathrm{pH} 7.4)$ to an absorbance of $0.70 \pm 0.02$ at $734 \mathrm{~nm}$. After that, $5 \mu \mathrm{L}$ of each sample was added to $295 \mu \mathrm{L}$ of diluted ABTS ${ }^{*+}$ solution in 96 well cuvettes. The mixture was kept in the dark for $10 \mathrm{~min}$ and detected at $734 \mathrm{~nm}$, using a microplate reader. Ethanol and Trolox were used as a negative and positive control, respectively. All determinations were performed in duplicate. 
The SORS of all considered samples has been investigated using the method described by Li and co-workers [28]. Briefly, $5 \mu \mathrm{L}$ of the sample solution was mixed with $200 \mu \mathrm{L}$ of 2-amino-2-hydroxymethyl-propane-1,3-diol (Tris-HCl) buffer $(20 \mathrm{mM}, \mathrm{pH} 8)$ in 96 well cuvettes. Then proper aliquots from followed solution were added: $30 \mu \mathrm{L}$ of $1.8 \mathrm{mM}$ nicotinamide adenine dinucleotide (NADH), $30 \mu \mathrm{L}$ of $3.6 \mathrm{mM}$ nitro blue tetrazolium (NBT) and $40 \mu \mathrm{L} 1.34 \mathrm{mM}$ phenazine methosulfate (PMS). The mixture was left to react for 5 min at $25^{\circ} \mathrm{C}$, following the detection at $560 \mathrm{~nm}$ for each sample by means of a microplate reader. Ethanol and quercetin were used as a negative and positive control, respectively. All determinations were performed in duplicate.

The NO scavenging capacity was determined according to the method described by Streejayan and Rao [29]. Griess reagent was freshly prepared as a mixture of equal volumes of sulfanilic acid solution with a-naphthylamine solution. For the preparation of sulfanilic acid solution $0.3 \mathrm{~g}$ of sulfanilic acid was dissolved in $10 \mathrm{~mL}$ glacial acetic acid and $5 \mathrm{~mL}$ distilled water by heating on a water bath. After cooling, $10 \mathrm{~mL}$ of $10 \%(\mathrm{~m} / \mathrm{V})$ sodium chloride was added, and the solution was diluted to $50 \mathrm{~mL}$ with distilled water. The $\alpha$-naphthylamine solution was prepared by dissolving $0.015 \mathrm{~g}$ of $\alpha$-naphthylamine hydrochloride in $5 \mathrm{~mL}$ hot water. After cooling, $10 \mathrm{~mL}$ glacial acetic acid was added, and the resulted solution was diluted to $50 \mathrm{~mL}$ with distilled water. A volume of $90 \mu \mathrm{L}$ of $15 \mathrm{mM}$ sodium nitroprusside in $7.4 \mathrm{pH}$ phosphate buffered was mixed with $60 \mu \mathrm{L}$ of the sample in 96 well cuvettes and incubated at $25^{\circ} \mathrm{C}$ for $120 \mathrm{~min}$. Then, $150 \mu \mathrm{L}$ of freshly prepared Griess reagent was added and left to react for $25 \mathrm{~min}$. The detection was carried out at $540 \mathrm{~nm}$ using a microplate reader. Ethanol and quercetin were used as a negative and positive control, respectively. All determinations were performed in duplicate.

\section{Reducing antioxidant power}

The FRAP of the samples was determined using the method developed by Benzie and Strain [30]. In brief, the FRAP reagent was freshly prepared by mixing acetate buffer ( $300 \mathrm{mM}, \mathrm{pH} 3.6)$, a solution of $10 \mathrm{mM} \mathrm{2,4,6-tripyridyltriazine}$ (TPTZ) in $40 \mathrm{mM} \mathrm{HCl}$, and $20 \mathrm{mM} \mathrm{FeCl}_{3}$ at 25:1:1 (v/v/v). A volume of $295 \mu \mathrm{L}$ of FRAP reagent and $5 \mu \mathrm{L}$ of sample solution were added in 96 well cuvettes and mixed thoroughly. The absorbance was taken at $593 \mathrm{~nm}$ after incubation for $30 \mathrm{~min}$ with a microplate reader. Ethanol and quercetin were used as a negative and positive control, respectively. All determinations were performed in duplicate.

The CUPRAC was determined using the method proposed by Apak et al. [31]. For this method, a solution consisting of $2 \mathrm{mM}$ neocuproine, $1 \mathrm{mM} \mathrm{CuSO} \cdot 5 \mathrm{H}_{2} \mathrm{O}$ and $1 \mathrm{M}$ ammonium acetate was prepared and incubated for $5 \mathrm{~min}$ for the bis(neocuproine) copper (II) complex to be formed. An aliquot 
of $200 \mu \mathrm{L}$ of this solution was mixed with $95 \mu \mathrm{L}$ ultrapure water and a $5 \mu \mathrm{L}$ of sample and placed in 96 well cuvettes. The absorbance was taken at $450 \mathrm{~nm}$ with a microplate reader. Ethanol and quercetin were used as a negative and positive control, respectively. All determinations were performed in duplicate.

The CHROMAC assay followed the method applied by Işık and co-workers [32]. With respect to this, $200 \mu \mathrm{L}$ of $100 \mathrm{mg} / \mathrm{L} \mathrm{K}_{2} \mathrm{Cr}_{2} \mathrm{O}_{7}$ dissolved in a $50 \mathrm{mM}$ phosphate buffer $\mathrm{pH} 2.8$ was mixed with $50 \mu \mathrm{L}$ of sample and incubated for $5 \mathrm{~min}$ in a 96 wells cuvette. Afterward, $50 \mu \mathrm{L}$ of $0.5 \mathrm{mM} \mathrm{1,5-diphenylcarbazide}$ was added and the absorbance was taken with a microplate reader at $540 \mathrm{~nm}$. Ethanol and quercetin were used as a negative and positive control, respectively. All determinations were performed in duplicate.

The ability of biogenic amines and related drugs to chelate metal ions has been studied using the method developed by Dinis et al. [33]. Firstly, $5 \mu \mathrm{L}$ of samples were mixed with $200 \mu \mathrm{L}$ water and $40 \mu \mathrm{L} 0.375 \mathrm{mM}$ Mohr's Salt $\left(\left(\mathrm{NH}_{4}\right)_{2} \mathrm{Fe}\left(\mathrm{SO}_{4}\right)_{2} 6 \mathrm{H}_{2} \mathrm{O}\right)$ in 96 well cuvettes. The reaction mixture was incubated at room temperature for 5 minutes. After this, an aliquot of $40 \mu \mathrm{L}$ of $3.75 \mathrm{mM}$ ferrozine solution was added to the mixture and homogenized and the absorbance was recorded at 546 by means of a microplate reader. Ethanol and ethylenediaminetetraacetic acid (EDTA) were used as a negative and positive control, respectively. All determinations were performed in duplicate.

The antioxidant and chelating capacity were expressed in \% and were calculated using formula (1) for determination that implies color consumption and formula (2) for assays that involve color formation (FRAP, CUPRAC and CHROMAC):

$$
\begin{aligned}
& (\%)=\frac{\text { Anegative control-Asample }}{\text { Anegative control }} \times 100 \text { (1) } \\
& (\%)=\frac{\text { Asample-Anegative control }}{\text { Apositive control }} \times 100 \text { (2) }
\end{aligned}
$$

\section{Chemometrics}

Cluster analysis allows meaningful generalizations to be made about large quantities of data by recognizing among them a few basic patterns. It plays a key role in searching for structures in data. Each of these structures is called cluster or class. A class is a group of individuals (samples) which resemble each other more strongly, in terms of properties, than they resemble members of other classes. Generally, two types of algorithm are distinguished, these being hierarchical and non-hierarchical or relocation clustering. Both methods require the calculation of a (dis)similarity matrix. This (dis)similarity 
which is really a measure of the proximity of the pair of objects (points) in the p-dimensional characteristic space, defined by the p properties measured for each individual, is usually expressed in terms of either the Euclidean or the Mahalanobis distance between the two points. Two approaches are known to hierarchical clustering, these being agglomerative and divisive procedures. An agglomerative hierarchical clustering approach places each object in its own cluster and gradually merges these atomic clusters into larger and larger clusters until all objects are in a single cluster. Divisive hierarchical clustering reverses the process by starting with all objects in one cluster and subdividing it into smaller ones until, finally, each object is again in a cluster of its own. The number of clusters to be generated can be specified in advance, or it may be optimized by the algorithm itself according to certain criteria.

Cluster imaging applied also in this study is a modification of cluster analysis. Whereas cluster analysis is useful for grouping samples or variables, in cluster imaging (also called two-way joining), samples and variables are clustering simultaneously. A two-way hierarchical cluster analysis with visual pairs of data sets is accomplished. This approach can provide extensive information and details on data structure and variability when both samples and variables are suspected to contribute simultaneously to the uncovering of meaningful patterns of clusters [33].

Principal component analysis (PCA) is a favorite tool in chemometrics for data compression and information extraction. PCA finds linear combinations of the original measurement variables that describe the significant variations in the data. PCA is reducing the dimensionality of the original dataset by explaining the correlation amongst many variables in terms of a smaller number of underlying factors (principal components or PCs) without losing much information. The PCs are a very useful tool for examining the relationships between objects (samples), looking for groups and trends, sorting out outliers. The PCA and CA are unsupervised techniques which offer useful information about samples, but sometimes when the similarities are very prominent, they are not enough for a highly sustained conclusion [35].

All the graphs and classical chemometric methods namely HCA and PCA were performed using Statistica 8.0 (StatSoft, Inc. 1984-2007, Tulsa, USA).

The sum of ranking differences (SRD) compares methods not based on raw data but based on ranks. This chemometric method corresponds to the principle of parsimony and provides an easy tool to evaluate the methods: the smaller the sum, the better the method. The ranking differences are calculated based on the "average antioxidant activity" rank [36, 37]. The sum of ranking differences (SRD) method was applied, using the instructions provided in http://aki.ttk.mta.hu/srd/. The detailed description of the method can be found in the original publication of Károly Héberger [37]. 


\section{REFERENCES}

1. B. Halliwell; J.M.K. Gutteridge; Free Radicals in Biologie and Medicine, $4^{\text {th }}$ ed., Oxford University Press, Oxford, 2007.

2. J.M.K. Gutteridge; B. Halliwell; Biochem. Biophys. Res. Commun., 2010, 393, 561-564.

3. D.L. Gilbert; C.A. Colton; Reactive Oxygen Species in Biological Systems: An Interdisciplinary Approach. Kluwer Academic Publishers, New York, 2002.

4. E. Cadenas; L. Packer; Handbook of Antioxidants. $2^{\text {nd }}$ ed., Marcel Dekker, Inc., 2010.

5. L. Saso; O. Firuzi; Curr. Drug Targets, 2014, 15, 1-23.

6. H.E. Himwich; W.A. Himwich; Biogenic Amines, Elsevier, Amsterdam, 1964.

7. D.S. Goldstein, R. McCarty; T. Nagatsu; T. Mabeshima; Catecholamine Research: From Molecular Insights to Clinical Medicine, Kluwer Academic Publisher, New York, 2002.

8. S. Shioda; I. Homma; N. Kato; Transmitters and Modulators in Health and Disease, Springer, Tokyo, 2009.

9. M.A. Medina; J.L. Urdiales; C. Rodríguez-Caso; F.J. Ramírez; F. SánchezJiménez; Crit. Rev. Biochem. Mol. Biol., 2003, 38, 23-59.

10. B.J. Mans; J.M.C. Ribeiro; J.F. Andersen; J. Biol. Chem., 2008, 283, 1872118733.

11. T. Miura; S. Muraoka; T. Ogiso; Biochem. Pharmacol., 1998, 55, 2001-2006.

12. I. Gülçin; Chem. Biol. Int., 2009, 179, 71-80.

13. T. Kawashima, K. Ohkubo, S. Fukuzumi, J. Phys. Chem. B, 2010,114, 675-680.

14. H.Y. Zhang; Quant. Struct-Act. Relat., 2000, 19, 50-53.

15. H.Y. Zhang; Y.M. Sun; X. L. Wang; Chem. Eur. J., 2003, 9, 502-508.

16. H.Y. Zhang; Curr. Comput-Aided Drug. Des., 2005, 1, 257-273.

17. Y. Da-Peng; J. Hong-Fang; T. Guang-Yan; R. Wei; Z. Hong-Yu; Molecules, 2007, 12, 878-884.

18. F. Shahidi; Natural Antioxidants: Chemistry, Health Effects, and Applications, AOCS Press, 1997.

19. C.E. Thomas; Handbook of Synthetic Antioxidants, Marcel Dekker, Inc., New York, 1997.

20. Comprehensive Medicinal Chemistry, Release 2004.1; MDL Information Systems Inc., San Leandro, CA, 2004.

21. K. Robards; P.D. Prenzler; G. Tucker; P. Swatsitang; W. Glover; Food Chem., 1999, 66, 401-436.

22. R. Apak; E. Capanoglu; F. Shahidi; Editors, Measurement of Antioxidant Activity \& Capacity: Recent Trends and Applications, $1^{\text {st }}$ ed., John Wiley \& Sons Ltd., Chichester, 2018.

23. C. Sârbu, D. Casoni; Cent. Eur. J. Chem., 2013, 11, 679-688.

24. D. Casoni; C. Sârbu; Spectrochim. Acta A, 2014, 118, 343-348. 
25. A. Guidea; C. Zăgrean-Tuza; A.C. Moț; C. Sârbu; Spectrochim. Acta A, 2020, 233, 118158.

26. M.S. Blois; Nature, 1958, 181, 1199-1200.

27. P. Re; N. Pellegrini; A. Proteggente; A. Pannala; M. Yang; C. Rice-Evans; Free Radic. Biol. Med., 1999, 26, 1231-1237.

28. X.L. Li; A.G. Zhou; Y. Han; Y. Carbohydr. Polym., 2006, 66, 34-42.

29. M.N.A. Rao; J. Pharm. Pharmacol. 1997, 49, 105-107.

30. I.F. Benzie; J.J. Strain; Anal. Biochem., 1996, 239, 70-76.

31. R. Apak; K. Güçlü; B. Demirata; et al. Molecules, 2007, 12, 1496-1547.

32. E. Işık; S. Şahin; C. Demir; Talanta, 2013, 111, 119-124.

33. T.C.P. Dinis; V.M.C. Madeira; L.M. Almeida; Arch. Biochem. Biophys., 1994, 315, 161-169.

34. L. Wilkinson; M. Friendly; Am. Stat., 2009, 63, 179-184.

35. O. Horovitz; C. Sârbu; H.F. Pop; Clasificarea raţională a elementelor chimice, Editura Dacia, Cluj-Napoca, 2000.

36. K. Héberger; Trac. Trends Anal. Chem., 2010, 29, 101-109.

37. K. Héberger; K. Kollár-Hunek; J. Chemom., 2011, 25, 151-158. 
